\title{
HEDGING OF GAME OPTIONS IN DISCRETE MARKETS WITH TRANSACTION COSTS
}

\author{
YURI KIFER \\ INSTITUTE OF MATHEMATICS \\ HEBREW UNIVERSITY \\ JERUSALEM, ISRAEL
}

\begin{abstract}
We construct algorithms for computation of prices and superhedging strategies for game options in general discrete time markets with transaction costs both from seller's (upper arbitrage free price) and buyer's (lower arbitrage free price) points of view.
\end{abstract}

\section{INTRODUCTION}

Game options introduced in [6] were studied by now in scores of papers but their investigation for markets with transaction costs remains on its initial stages (see [3] and [4]). In this paper we extend to the game options case the theory of pricing and hedging of options in general discrete markets with proportional transaction costs in the form which was previously developed for American options case in [2], 1] and [8].

It is well known that pricing in markets with transaction costs becomes somewhat similar to pricing in incomplete markets so that hedging arguments from the option seller's and buyer's point of view lead to different prices which determine the whole range of arbitrage free prices. An interesting but not surprising feature of game options is the almost complete symmetry between the seller's and the buyer's pricing approaches which will be demonstrated clearly in the statements of results and their proofs in this paper.

We will derive representations both for upper (ask, seller's) and lower (bid, buyer's) hedging prices of game options with transaction costs and will exhibit dynamical programming type algorithms for their computation, as well, as for computation of corresponding seller's and buyer's superhedging strategies. We demonstrate for game options only analogues of some of representations and algorithms from [8] concentrating on those which allow symmetric expositions of seller's and buyer's cases. Others can be obtained in a similar way and their inclusion here would make this paper too overloaded and more difficult to read.

Superhedging requires an option seller to invest a large sum into his hedging portfolio and in some circumstances he may prefer to accept some risk setting up a portfolio with smaller initial amount. In fact, we will define in Section 5 the shortfall risk both for the seller and the buyer showing that also in the study of the risk for game options the symmetry between these two market participants can be preserved. Dynamical programming type algorithms for computation of shortfall risks and corresponding partial hedging strategies in the setup of game options with transaction costs can be obtained similarly to [3] where they were derived for American options in binomial markets with the motivation to approximate the shortfall risk of the continuous time Black-Scholes model.

Date: November 7, 2018.

2000 Mathematics Subject Classification. Primary 91B28: Secondary: 60G40, 91B30 .

Key words and phrases. game options, transaction costs, superhedging, shortfall risk, Dynkin games .

Partially supported by the ISF grant no. 82/10. 


\section{Preliminaries}

We will deal with the same market model as in 8 which consists of a finite probability space $\Omega$ with the $\sigma$-field $\mathcal{F}=2^{\Omega}$ of all subspaces of $\Omega$ and a probability measure $\mathbb{P}$ on $\mathcal{F}$ giving a positive weight $\mathbb{P}(\omega)$ to each $\omega \in \Omega$. The setup includes also a filtration $\{\emptyset, \Omega\}=\mathcal{F}_{0} \subset \mathcal{F}_{1} \subset \ldots \subset \mathcal{F}_{T}=\mathcal{F}$ where $T$ is a positive integer called the time horizon. It is convenient to denote by $\Omega_{t}$ the set of atoms in $\mathcal{F}_{t}$ so that any $\mathcal{F}_{t}$-measurable random variable (vector) $Z$ can be identified with a function (vector function) defined on $\Omega_{t}$ and its value at $\mu \in \Omega_{t}$ will be denoted either by $Z(\mu)$ or by $Z^{\mu}$. The points of $\Omega_{t}$ can be viewed as vertices of a tree so that an arrow is drawn from $\mu \in \Omega_{t}$ to $\nu \in \Omega_{t+1}$ if $\nu \subset \mu$.

The market model consists of a risk-free bond and a risky stock. Without loss of generality, we can assume that all prices are discounted so that the bond price equals 1 all the time and a position in bonds is identified with cash holding. On the other hand, the shares of the stock can be traded which involves proportional transaction costs. This will be represented by bid-ask spreads, i.e. shares can be bought at an ask price $S_{t}^{a}$ or sold at the bid price $S_{t}^{b}$, where $S_{t}^{s} \geq S_{t}^{b}>0, t=0,1, \ldots, T$ are processes adapted to the filtration $\left\{\mathcal{F}_{t}\right\}_{t=o}^{T}$.

The liquidation value at time $t$ of a portfolio $(\gamma, \delta)$ consisting of an amount $\gamma$ of cash (or bond) and $\delta$ shares of the stock equals

$$
\theta_{t}(\gamma, \delta)=\gamma+S_{t}^{b} \delta^{+}-S_{t}^{a} \delta^{-}
$$

which in case $\delta<0$ means that a potfolio owner should spend the amount $S_{t}^{a} \delta^{-}$in order to close his short position. Observe that fractional numbers of shares are allowed here so that both $\gamma$ and $\delta$ in a portfolio $(\gamma, \delta)$ could be, in priciple, any real numbers. By definition, a self-financing portfolio strategy is a predictable process $\left(\alpha_{t}, \beta_{t}\right)$ representing positions in cash (or bonds) and stock at time $t, t=0,1, \ldots, T$ such that

$$
\theta_{t}\left(\alpha_{t}-\alpha_{t+1}, \beta_{t}-\beta_{t+1}\right) \geq 0 \quad \forall t=0,1, \ldots, T-1
$$

and the set of all such portfolio strategies will be denoted by $\Phi$.

Recall that a game (or Israeli) option (contingent claim) introduced in [6 is defined as a contract between its seller and buyer such that both have the right to exercise it at any time up to a maturity date (horizon) $T$. If the buyer exercises the contract at time $t$, then he receives from the seller a payment $Y_{t}$ while if the latter exercises at time $t$ then his payment to the buyer becomes $X_{t} \geq Y_{t}$ and the difference $\Delta_{t}=X_{t}-Y_{t}$ is interpreted as a penalty for the contract cancellation. In the presence of transaction costs there is a difference whether we stipulate that the option to be settled in cash or both in cash and shares of stock while in the former case an assumption concerning transaction costs in the process of portfolio liquidation should be made. We adopt here the setup where the payments $X_{t}$ and $Y_{t}$ are made both in cash and in shares of the stock and transaction costs take place always when a portfolio adjustment occurs. Thus, the payments are, in fact, adapted random 2-vectors $X_{t}=\left(X_{t}^{(1)}, X_{t}^{(2)}\right)$ and $Y_{t}=\left(Y_{t}^{(1)}, Y_{t}^{(2)}\right)$ where the first and the second coordinates represent, respectively, a cash amount to be payed and a number of stock shares to be delivered and as we allow also fractional numbers of shares both coordinates can take on any nonnegative real value. The inequality $X_{t} \geq Y_{t}$ in the zero transaction costs case is replaced in our present setup by

$$
\Delta_{t}=\theta_{t}\left(X_{t}^{(1)}-Y_{t}^{(1)}, X_{t}^{(2)}-Y_{t}^{(2)}\right) \geq 0
$$

and $\Delta_{t}$ is interpreted as a cancellation penalty. We impose also a natural assumption that $X_{T}^{(1)}=Y_{T}^{(1)}$ and $X_{T}^{(2)}=Y_{T}^{(2)}$, i.e. on the maturity date there is no penalty. Therefore, if the seller cancells the contract at time $s$ while the buyer exercises at time $t$ the former delivers to the latter a package of cash and stock shares which can be represented as a 2 -vector in the form

$$
Q_{s, t}=\left(Q_{s, t}^{(1)}, Q_{s, t}^{(2)}\right)=X_{s} \mathbb{I}_{s<t}+Y_{t} \mathbb{I}_{t \leq s}
$$


where $\mathbb{I}_{A}=1$ if an event $A$ occurs and $\mathbb{I}_{A}=0$ if not. It will be convenient to allow the payment components $X_{t}^{(1)}, X_{t}^{(2)}$ and $Y_{t}^{(1)}, Y_{t}^{(2)}$ to take on any real (and not only nonnegative) values which will enable us to demonstrate complete duality (symmetry) between the seller's and the buyer's positions.

A pair $(\sigma, \pi)$ of a stopping time $\sigma \leq T$ and of a self-financing strategy $\pi=\left(\alpha_{t}, \beta_{t}\right)_{t=0}^{T}$ will be called a superhedging strategy for the seller of the game option with a payoff given by (2.4) if for all $t \leq T$,

$$
\theta_{\sigma \wedge t}\left(\alpha_{\sigma \wedge t}-Q_{\sigma, t}^{(1)}, \beta_{\sigma \wedge t}-Q_{\sigma, t}^{(2)}\right) \geq 0
$$

where, as usual, $c \wedge d=\min (c, d)$ and $c \vee d=\max (c, d)$. The seller's (ask or upper hedging) price $V^{a}$ of a game option is defined as the infimum of initial amounts required to start a superhedging strategy for the seller. Since in order to get $\alpha_{0}$ amount of cash and $\beta_{0}$ shares of stock at time 0 the seller should spend

$$
-\theta_{0}\left(-\alpha_{0},-\beta_{0}\right)=\alpha_{0}+\beta_{0}^{+} S_{0}^{a}-\beta_{0}^{-} S_{0}^{b}
$$

in cash, we can write

$$
V^{a}=\inf _{\sigma, \pi}\left\{-\theta_{0}\left(-\alpha_{0},-\beta_{0}\right):(\sigma, \pi) \text { with } \pi=\left(\alpha_{t}, \beta_{t}\right)_{t=0}^{T} \text { being a superhedging strategy for the seller }\right\} .
$$

On the other hand, the buyer may borrow from a bank an amount $\theta_{0}\left(-\alpha_{0},-\beta_{0}\right)$ to purchase a game option with the payoff (2.4) and starting with the negative valued portfolio $\left(\alpha_{0}, \beta_{0}\right)$ to manage a selffinancing strategy $\pi=\left(\alpha_{t}, \beta_{t}\right)_{t=0}^{T}$ so that for a given stopping time $\tau \leq T$ and all $s \leq T$,

$$
\theta_{s \wedge \tau}\left(\alpha_{s \wedge \tau}+Q_{s, \tau}^{(1)}, \beta_{s \wedge \tau}+Q_{s, \tau}^{(2)}\right) \geq 0 .
$$

In this case the pair $(\tau, \pi)$ will be called a superhedging strategy for the buyer. The buyer's (bid or lower hedging) price $V^{b}$ of the game option above is defined as the supremum of initial bank loan required to purchase this game option and to manage a superhedging strategy for the buyer. Thus,

$$
V^{b}=\sup _{\tau, \pi}\left\{\theta_{0}\left(-\alpha_{0},-\beta_{0}\right):(\tau, \pi) \text { with } \pi=\left(\alpha_{t}, \beta_{t}\right)_{t=0}^{T} \text { being a superhedging strategy for the buyer }\right\} .
$$

It follows from the representations of Theorem 3.1 below that $V^{a} \geq V^{b}$.

The goal of this paper is to obtain representations of $V^{a}$ and $V^{b}$ in the form of inf sup expressions and to construct backward and forward induction algorithms for computation both of these prices and of corresponding superhedging strategies. These results will be stated precisely in the next section. As in the case of American options with transaction costs in 2, 1 and $[8$ precise statements of our results involve the notion of randomized stopping times and approximate martingales which will be introduced in the next section.

\section{SupERHEDGING AND PRICE REPRESENTATIONS: STATEMENTS}

First, we recall the notion of a randomized stopping time (see 2, 1], 8] and references there) which is defined as a nonnegative adapted process $\chi$ such that $\sum_{t=0}^{T} \chi_{t}=1$. The set of all randomized stopping times will be denoted by $\mathcal{X}$ while the set of all usual or pure stopping times will be denoted by $\mathcal{T}$. It will be convenient to identify each pure stopping time $\tau$ with a randomized stopping time $\chi^{\tau}$ such that $\chi_{t}^{\tau}=\mathbb{I}_{\{\tau=t\}}$ for any $t=0,1, \ldots, T$, so that we could write $\mathcal{T} \subset \mathcal{X}$. For any adapted process $Z$ and each randomized stopping time $\chi$ the time- $\chi$ value of $Z$ is defined by

$$
Z_{\chi}=\sum_{t=0}^{T} \chi_{t} Z_{t} .
$$

Considering a game option with a payoff given by (2.4) we write also

$$
Q_{\chi, \tilde{\chi}}=\sum_{s, t=0}^{T} \chi_{s} \tilde{\chi}_{t} Q_{s, t}
$$


which is the seller's payment to the buyer when the former cancells and the latter exercises at randomized stopping times $\chi$ and $\tilde{\chi}$, respectively. In particular, if $\sigma$ and $\tau$ are pure stopping times then

$$
Q_{\chi, \chi^{\tau}}=\sum_{s=0}^{T} \chi_{s} Q_{s, \tau} \text { and } Q_{\chi^{\sigma}, \chi}=\sum_{t=0}^{T} \chi_{t} Q_{\sigma, t} .
$$

We can also define the "minimum" and the "maximum" of two randomized stopping times $\chi$ and $\tilde{\chi}$ which are randomized stopping times $\chi \wedge \tilde{\chi}$ and $\chi \vee \tilde{\chi}$ given by

$$
\begin{gathered}
(\chi \wedge \tilde{\chi})_{t}=\chi_{t} \sum_{s=t}^{T} \tilde{\chi}_{s}+\tilde{\chi}_{t} \sum_{s=t+1}^{T} \chi_{s} \text { and } \\
(\chi \vee \tilde{\chi})_{t}=\chi_{t} \sum_{s=0}^{t} \tilde{\chi}_{s}+\tilde{\chi}_{t} \sum_{s=0}^{t-1} \chi_{s} .
\end{gathered}
$$

In particular, if $\sigma$ and $\tau$ are pure stopping times then

$$
\chi^{\sigma} \wedge \chi^{\tau}=\chi^{\sigma \wedge \tau}, \chi^{\sigma} \vee \chi^{\tau}=\chi^{\sigma \vee \tau}
$$

and for any adapted process $Z$,

$$
Z_{\chi \wedge \chi^{\tau}}=\sum_{s=0}^{T} \chi_{s} Z_{s \wedge \tau}, Z_{\chi^{\sigma} \wedge \chi}=\sum_{t=0}^{T} \chi_{t} Z_{\sigma \wedge t}
$$

and similarly for $\chi \vee \chi^{\tau}$ and $\chi^{\sigma} \vee \chi$.

Next, we introduce the notion of an approximate martingale which is defined for any randomized stopping time $\chi$ as a pair $(P, S)$ of a probability measure $P$ on $\Omega$ and of an adapted process $S$ such that for each $t=0,1, \ldots, T$,

$$
S_{t}^{b} \leq S_{t} \leq S_{t}^{a} \text { and } \chi_{t+1}^{*} S_{t}^{b} \leq \mathbb{E}_{P}\left(S_{t+1}^{\chi^{*}} \mid \mathcal{F}_{t}\right) \leq \chi_{t+1}^{*} S_{t}^{a}
$$

where $\mathbb{E}_{P}$ is the expectation with respect to $P$,

$$
\chi_{t}^{*}=\sum_{s=t}^{T} \chi_{s}, Z_{t}^{\chi^{*}}=\sum_{s=t}^{T} \chi_{s} Z_{s}, \chi_{T+1}^{*}=0 \text { and } Z_{T+1}^{\chi^{*}}=0 .
$$

Given a randomized stopping time $\chi$ the space of corresponding approximate martingales $(P, S)$ will be denoted by $\overline{\mathcal{P}}(\chi)$ and we denote by $\mathcal{P}(\chi)$ the subspace of $\overline{\mathcal{P}}(\chi)$ consisting of pairs $(P, S)$ with $P$ being equivalent to the original (market) probability $\mathbb{P}$.

Now we can formulate some of our results which exhibit ask and bid price representations for game options.

Theorem 3.1. In the above notations,

$$
\begin{aligned}
V^{a} & =\min _{\sigma \in \mathcal{T}} \max _{\chi \in \mathcal{X}} \max _{(P, S) \in \overline{\mathcal{P}}(\chi)} \mathbb{E}_{P}\left(Q_{\sigma, \cdot}^{(1)}+S Q_{\sigma, \cdot}^{(2)}\right)_{\chi} \\
& =\min _{\sigma \in \mathcal{T}} \max _{\chi \in \mathcal{X}} \sup _{(P, S) \in \mathcal{P}(\chi)} \mathbb{E}_{P}\left(Q_{\sigma, \cdot}^{(1)}+S Q_{\sigma, \cdot}^{(2)}\right)_{\chi}
\end{aligned}
$$

and

$$
\begin{aligned}
V^{b} & =\max _{\tau \in \mathcal{T}} \min _{\chi \in \mathcal{X}} \min _{(P, S) \in \overline{\mathcal{P}}(\chi)} \mathbb{E}_{P}\left(Q_{\cdot, \tau}^{(1)}+S Q_{\cdot, \tau}^{(2)}\right)_{\chi} \\
& =\max _{\tau \in \mathcal{T}} \min _{\chi \in \mathcal{X}} \inf _{(P, S) \in \mathcal{P}(\chi)} \mathbb{E}_{P}\left(Q_{\cdot, \tau}^{(1)}+S Q_{\cdot, \tau}^{(2)}\right)_{\chi}
\end{aligned}
$$

where $Q_{\sigma, \cdot}^{(1)}, Q_{\sigma, \cdot}^{(2)}$ and $Q_{\cdot, \tau}^{(1)}, Q_{\cdot, \tau}^{(2)}$ denote functions on $\{0,1, \ldots, T\}$ whose values at $t$ are obtained by replacing $\cdot$ by $t$.

In order to exhibit dynamical programming algorithms for computation of $V^{a}$ and $V^{b}$ and induction algorithms producing corresponding superhedging strategies we have to introduce first some convex analysis notions and notations (see [7] and [8] for more details). Denote by $\Theta$ the family of functions $f: \mathbb{R} \rightarrow \mathbb{R} \cup\{-\infty\}$ such that either $f \equiv-\infty$ or $f$ is a (finite) real valued polyhedral (continuous piecewise linear with finite number of segments) function. If $f, g \in \Theta$ then, clearly, $f \wedge g, f \vee g \in \Theta$. The epigraph of 
$f \in \Theta$ is defined by epi $(f)=\left\{(x, y) \in \mathbb{R}^{2}: x \geq f(y)\right\}$. For any $c \geq d$ the function $h_{[d, c]}(y)=c y^{-}-d y^{+}$, clearly, belongs to $\Theta$. Observe that the self-financing condition (2.2) can be rewritten in the form

$$
\left(\alpha_{t}-\alpha_{t+1}, \beta_{t}-\beta_{t+1}\right) \in \operatorname{epi}\left(h_{\left[S_{t}^{b}, S_{t}^{a}\right]}\right) .
$$

For each $f \in \Theta$ and $c \geq d$ there exists a unique function $\operatorname{gr}_{[d, c]}(f) \in \Theta$ such that

$$
\operatorname{epi}\left(\operatorname{gr}_{[d, c]}(f)\right)=\operatorname{epi}\left(h_{[d, c]}\right)+\operatorname{epi}(f) \text {. }
$$

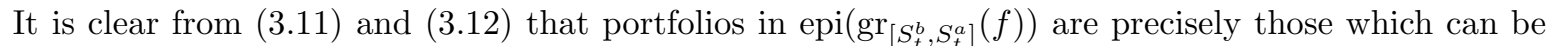
rebalanced in a self-financing manner at time $t$ to yield a portfolio in epi $(f)$. Denote by $\Lambda$ the family of all convex functions in $\Theta$ and by $\Gamma$ the family of concave functions $v: \mathbb{R} \rightarrow \mathbb{R} \cup\{-\infty\}$ which are polyhedral on their essential domain $\operatorname{dom}(v)=\{x \in \mathbb{R}: v(x)>-\infty\}$. For any $f \in \Lambda$ the convex duality sais that

$$
f^{*}(x)=\inf _{y \in \mathbb{R}}(f(y)+x y) \in \Gamma \text { and } f(y)=\sup _{x \in \mathbb{R}}\left(f^{*}(x)-x y\right),
$$

the infimum and the supremum above are attained whenever they are finite.

For any $y \in \mathbb{R}, \mu \in \Omega_{t}$ and $t=0,1, \ldots, T$ define $q_{t}^{a}(y)=q_{t}^{a}(\mu, y), q_{t}^{b}(y)=q_{t}^{b}(\mu, y), r_{t}^{a}(y)=r_{t}^{a}(\mu, y)$, $r_{t}^{b}(y)=r_{t}^{b}(\mu, y)$ by

$$
\begin{gathered}
q_{t}^{a}(y)=X_{t}^{(1)}+h_{\left[S_{t}^{b}, S_{t}^{a}\right]}\left(y-X_{t}^{(2)}\right), r_{t}^{a}(y)=Y_{t}^{(1)}+h_{\left[S_{t}^{b}, S_{t}^{a}\right]}\left(y-Y_{t}^{(2)}\right) \\
q_{t}^{b}(y)=-X_{t}^{(1)}+h_{\left[S_{t}^{b}, S_{t}^{a}\right]}\left(y+X_{t}^{(2)}\right), r_{t}^{b}(y)=-Y_{t}^{(1)}+h_{\left[S_{t}^{b}, S_{t}^{a}\right]}\left(y+Y_{t}^{(2)}\right)
\end{gathered}
$$

with $h_{[d, c]}$ the same as in (3.11) and (3.12). Observe that if $c \geq d \geq 0$ then either $h_{[d, c]} \equiv 0$ or $h_{[d, c]}$ is a monotone decreasing function, and so

$$
q_{t}^{a} \geq r_{t}^{a} \text { and } q_{t}^{b} \leq r_{t}^{b}
$$

Introduce also

$$
\begin{gathered}
G_{s, t}(y)=Q_{s, t}^{(1)}+h_{\left[S_{s \wedge t}^{b}, S_{s \wedge t}^{a}\right]}\left(y-Q_{s, t}^{(2)}\right)=q_{s}^{a}(y) \mathbb{I}_{s<t}+r_{t}^{a}(y) \mathbb{I}_{t \leq s} \text { and } \\
H_{s, t}(y)=-Q_{s, t}^{(1)}+h_{\left[S_{s \wedge t}^{b}, S_{s \wedge t}^{a}\right]}\left(y+Q_{s, t}^{(2)}\right)=q_{s}^{b}(y) \mathbb{I}_{s<t}+r_{t}^{b}(y) \mathbb{I}_{t \leq s} .
\end{gathered}
$$

Clearly, the superhedging conditions (2.5) of the seller and (2.8) of the buyer are equivalent to

$$
\begin{gathered}
\left(\alpha_{\sigma \wedge t}, \beta_{\sigma \wedge t}\right) \in \operatorname{epi}\left(G_{\sigma, t}\right) \text { for all } t=0,1, \ldots, T \text { and } \\
\left(\alpha_{s \wedge \tau}, \beta_{s \wedge \tau}\right) \in \operatorname{epi}\left(H_{s, \tau}\right) \text { for all } s=0,1, \ldots, T,
\end{gathered}
$$

respectively. Observe also that

$$
q_{t}^{a}(0)=-q_{t}^{b}(0)=\theta_{t}\left(X_{t}^{(1)}, X_{t}^{(2)}\right) \text { and } r_{t}^{a}(0)=-r_{t}^{b}(0)=\theta_{t}\left(Y_{t}^{(1)}, Y_{t}^{(2)}\right) .
$$

We recall that $X_{T}^{(1)}=Y_{T}^{(1)}$ and $X_{T}^{(2)}=Y_{T}^{(2)}$, and so $q_{T}^{a}=r_{T}^{a}$ and $q_{T}^{b}=r_{T}^{b}$. The following result provides dynamical programming algorithms for ask and bid prices computations.

Theorem 3.2. (i) For any $x \in \mathbb{R}, \mu \in \Omega_{T}$ and $\sigma \in \mathcal{T}$ define

$$
z_{T}^{\mu}(x)=w_{T}^{\mu}(x)=r_{T}^{a}(\mu, x) \text { and } z_{\sigma, T}^{\mu}(x)=w_{\sigma, T}^{\mu}(x)=G_{\sigma, T}(\mu, x) .
$$

Next, for $t=1,2, \ldots, T$ and each $\mu \in \Omega_{t-1}$ define by backward induction

$$
\begin{gathered}
\mathbf{z}_{t-1}^{\mu}=\max _{\nu \subset \mu, \nu \in \Omega_{t}} z_{t}^{\nu}, \mathbf{z}_{\sigma, t-1}^{\mu}=\max _{\nu \subset \mu, \nu \in \Omega_{t}} z_{\sigma, t}^{\nu}, \\
w_{t-1}^{\mu}=g r_{\left[S_{t-1}^{b}(\mu), S_{t-1}^{a}(\mu)\right]}\left(\mathbf{z}_{t-1}^{\mu}\right), w_{\sigma, t-1}^{\mu}=g r_{\left[S_{t-1}^{b}(\mu), S_{t-1}^{a}(\mu)\right]}\left(\mathbf{z}_{\sigma, t-1}^{\mu}\right), \\
z_{t-1}^{\mu}(x)=\min \left(q_{t-1}^{a}(\mu, x), \max \left(r_{t-1}^{a}(\mu, x), w_{t-1}^{\mu}(x)\right)\right) \text { and } z_{\sigma, t-1}^{\mu}(x)=\max \left(G_{\sigma, t-1}(\mu, x), w_{\sigma, t-1}^{\mu}(x)\right) .
\end{gathered}
$$

Then $z_{0}(0)=\min _{\sigma \in \mathcal{T}} z_{\sigma, 0}(0)=V^{a}$.

(ii) For any $x \in \mathbb{R}, \mu \in \Omega_{T}$ and $\tau \in \mathcal{T}$ define

$$
u_{T}^{\mu}(x)=v_{T}^{\mu}(x)=r_{T}^{b}(\mu, x) \text { and } u_{T, \tau}^{\mu}(x)=v_{T, \tau}^{\mu}(x)=H_{T, \tau}(\mu, x) .
$$


Next, for $t=1,2, \ldots, T$ and each $\mu \in \Omega_{t-1}$ define by the backward induction

$$
\begin{gathered}
\mathbf{u}_{t-1}^{\mu}=\max _{\nu \subset \mu, \nu \in \Omega_{t}} u_{t}^{\nu}, \mathbf{u}_{t-1, \tau}^{\mu}=\max _{\nu \subset \mu, \nu \in \Omega_{t}} u_{t, \tau}^{\nu}, \\
v_{t-1}^{\mu}=g r_{\left[S_{t-1}^{b}(\mu), S_{t-1}^{a}(\mu)\right]}\left(\mathbf{u}_{t-1}^{\mu}\right), v_{t-1, \tau}^{\mu}=g r_{\left[S_{t-1}^{b}(\mu), S_{t-1}^{a}(\mu)\right]}\left(\mathbf{u}_{t-1, \tau}^{\mu}\right), \\
u_{t-1}^{\mu}(x)=\min \left(r_{t-1}^{b}(\mu, x), \max \left(q_{t-1}^{b}(\mu, x), v_{t-1}^{\mu}(x)\right)\right) \text { and } u_{t-1, \tau}^{\mu}(x)=\max \left(H_{t-1, \tau}(\mu, x), v_{t-1, \tau}^{\mu}(x)\right) .
\end{gathered}
$$

Then $u_{0}(0)=\max _{\tau \in \mathcal{T}} u_{0, \tau}(0)=-V^{b}$.

Next, we describe inductive constructions of superhedging seller's and buyer's strategies using the functions $z_{t}$ and $u_{t}, t=0, \ldots, T$ constructed in Theorem 3.2

Theorem 3.3. (i) Construct by induction a sequence of (pure) stopping times $\sigma_{t} \in \mathcal{T}$ and a self-financing strategy $(\alpha, \beta)$ such that

$$
\left(\alpha_{t}, \beta_{t}\right) \in \operatorname{epi}\left(z_{t}\right) \backslash \operatorname{epi}\left(q_{t}^{a}\right) \text { on }\left\{t<\sigma_{t}\right\}
$$

for each $t=0,1, \ldots, T$ in the following way. First, take any $\mathcal{F}_{0}$-measurable portfolio $\left(\alpha_{0}, \beta_{0}\right) \in$ epi $\left(z_{0}\right)$ and set

$$
\sigma_{0}= \begin{cases}0 & \text { if }\left(\alpha_{0}, \beta_{0}\right) \in \operatorname{epi}\left(q_{0}^{a}\right) \\ T & \text { if }\left(\alpha_{0}, \beta_{0}\right) \notin \operatorname{epi}\left(q_{0}^{a}\right) .\end{cases}
$$

Suppose that an $\mathcal{F}_{t}$-measurable portfolio $\left(\alpha_{t}, \beta_{t}\right) \in$ epi $\left(z_{t}\right)$ and a stopping time $\sigma_{t} \in \mathcal{T}$ have already been constructed for some $t=0,1, \ldots T-1$ so that (3.23) holds true. By (3.12) and (3.20),

$$
\left(\alpha_{t}, \beta_{t}\right) \in \operatorname{epi}\left(w_{t}\right)=\operatorname{epi}\left(h_{\left[S_{t}^{b}, S_{t}^{a}\right]}\right)+\operatorname{epi}\left(\mathbf{z}_{t}\right) \text { on }\left\{t<\sigma_{t}\right\},
$$

and so there exists an $\mathcal{F}_{t}$-measurable portfolio $\left(\alpha_{t+1}, \beta_{t+1}\right)$ such that

$$
\left(\alpha_{t+1}, \beta_{t+1}\right) \in \operatorname{epi}\left(\mathbf{z}_{t}\right),\left(\alpha_{t}-\alpha_{t+1}, \beta_{t}-\beta_{t+1}\right) \in \operatorname{epi}\left(h_{\left[S_{t}^{b}, S_{t}^{a}\right]}\right) \text { on }\left\{t<\sigma_{t}\right\}
$$

and $\left(\alpha_{t+1}, \beta_{t+1}\right)=\left(\alpha_{t}, \beta_{t}\right)$ on $\left\{t \geq \sigma_{t}\right\}$ which provides the self-financing condition (3.11) both on $\left\{t<\sigma_{t}\right\}$ and on $\left\{t \geq \sigma_{t}\right\}$. By (3.20) it follows also that $\left(\alpha_{t+1}, \beta_{t+1}\right) \in \operatorname{epi}\left(z_{t+1}\right)$ on $\left\{t<\sigma_{t}\right\} \supset\left\{t+1<\sigma_{t+1}\right\}$. Set

$$
\sigma_{t+1}= \begin{cases}\sigma_{t} & \text { if } t \geq \sigma_{t} \\ t+1 & \text { if } t<\sigma_{t} \text { and }\left(\alpha_{t+1}, \beta_{t+1}\right) \in \operatorname{epi}\left(q_{t+1}^{a}\right) \\ T & \text { if } t<\sigma_{t} \text { and }\left(\alpha_{t+1}, \beta_{t+1}\right) \notin \operatorname{epi}\left(q_{t+1}^{a}\right) .\end{cases}
$$

Finally, set $\sigma=\sigma_{T} \in \mathcal{T}$. Then the pair $(\sigma, \pi)$ with $\pi=(\alpha, \beta)$ constructed by the above algorithm with $\left(\alpha_{0}, \beta_{0}\right)=\left(V^{a}, 0\right)$ is a superhedging strategy for the seller.

(ii) Construct by induction a sequence of (pure) stopping times $\tau_{t} \in \mathcal{T}$ and a self-financing strategy $(\alpha, \beta)$ such that

$$
\left(\alpha_{t}, \beta_{t}\right) \in \operatorname{epi}\left(u_{t}\right) \backslash \operatorname{epi}\left(r_{t}^{b}\right) \text { on }\left\{t<\tau_{t}\right\}
$$

for each $t=0,1, \ldots, T$ in the following way. First, take any $\mathcal{F}_{0}$-measurable portfolio $\left(\alpha_{0}, \beta_{0}\right) \in$ epi $\left(u_{0}\right)$ and set

$$
\tau_{0}= \begin{cases}0 & \text { if }\left(\alpha_{0}, \beta_{0}\right) \in \operatorname{epi}\left(r_{0}^{b}\right) \\ T & \text { if }\left(\alpha_{0}, \beta_{0}\right) \notin \operatorname{epi}\left(r_{0}^{b}\right) .\end{cases}
$$

Suppose that an $\mathcal{F}_{t}$-measurable portfolio $\left(\alpha_{t}, \beta_{t}\right) \in$ epi $\left(u_{t}\right)$ and a stopping time $\tau_{t} \in \mathcal{T}$ have already been constructed for some $t=0,1, \ldots T-1$ so that (3.23) holds true. By (3.12) and (3.22),

$$
\left(\alpha_{t}, \beta_{t}\right) \in \operatorname{epi}\left(v_{t}\right)=\operatorname{epi}\left(h_{\left[S_{t}^{b}, S_{t}^{a}\right]}\right)+\operatorname{epi}\left(\mathbf{u}_{t}\right) \text { on }\left\{t<\tau_{t}\right\},
$$

and so there exists an $\mathcal{F}_{t}$-measurable portfolio $\left(\alpha_{t+1}, \beta_{t+1}\right)$ such that

$$
\left(\alpha_{t+1}, \beta_{t+1}\right) \in \operatorname{epi}\left(\mathbf{u}_{t}\right),\left(\alpha_{t}-\alpha_{t+1}, \beta_{t}-\beta_{t+1}\right) \in \operatorname{epi}\left(h_{\left[S_{t}^{b}, S_{t}^{a}\right]}\right) \text { on }\left\{t<\tau_{t}\right\}
$$


and $\left(\alpha_{t+1}, \beta_{t+1}\right)=\left(\alpha_{t}, \beta_{t}\right)$ on $\left\{t \geq \tau_{t}\right\}$ which provides the self-financing condition (3.11) both on $\left\{t<\tau_{t}\right\}$ and on $\left\{t \geq \tau_{t}\right\}$. By (3.22) it follows also that $\left(\alpha_{t+1}, \beta_{t+1}\right) \in$ epi $\left(u_{t+1}\right)$ on $\left\{t<\tau_{t}\right\} \supset\left\{t+1<\tau_{t+1}\right\}$. Set

$$
\tau_{t+1}= \begin{cases}\tau_{t} & \text { if } t \geq \tau_{t} \\ t+1 & \text { if } t<\tau_{t} \text { and }\left(\alpha_{t+1}, \beta_{t+1}\right) \in \operatorname{epi}\left(r_{t+1}^{b}\right) \\ T & \text { if } t<\tau_{t} \text { and }\left(\alpha_{t+1}, \beta_{t+1}\right) \notin \operatorname{epi}\left(r_{t+1}^{b}\right) .\end{cases}
$$

Finally, set $\tau=\tau_{T} \in \mathcal{T}$. Then the pair $(\tau, \pi)$ with $\pi=(\alpha, \beta)$ constructed by the above algorithm with $\left(\alpha_{0}, \beta_{0}\right)=\left(-V^{b}, 0\right)$ is a superhedging strategy for the buyer.

\section{Superhedging And PRICE Representations: Proofs}

We start with the following result.

Lemma 4.1. (i) The following assertions are equivalent:

(a) $(\gamma, \delta) \in \operatorname{epi}\left(z_{0}\right)$;

(b) There exists a self-financing strategy $(\alpha, \beta) \in \Phi$ and a stopping time $\sigma \in \mathcal{T}$ such that $\left(\alpha_{0}, \beta_{0}\right)=$ $(\gamma, \delta)$ and (3.16) holds true;

(c) There exists a superhedging strategy $(\sigma, \pi), \sigma \in \mathcal{T}, \pi=\left(\alpha_{t}, \beta_{t}\right)_{t=0}^{T} \in \Phi$ for the seller such that $\left(\alpha_{0}, \beta_{0}\right)=(\gamma, \delta)$.

(ii) The following assertions are equivalent:

(d) $(\gamma, \delta) \in \operatorname{epi}\left(u_{0}\right)$;

(e) There exists a self-financing strategy $(\alpha, \beta) \in \Phi$ and a stopping time $\tau \in \mathcal{T}$ such that $\left(\alpha_{0}, \beta_{0}\right)=$ $(\gamma, \delta)$ and (3.17) holds true;

(f) There exists a superhedging strategy $(\tau, \pi), \tau \in \mathcal{T}, \pi=\left(\alpha_{t}, \beta_{t}\right)_{t=0}^{T} \in \Phi$ for the buyer such that $\left(\alpha_{0}, \beta_{0}\right)=(\gamma, \delta)$.

Proof. (i) Suppose that $(\gamma, \delta) \in \operatorname{epi}\left(z_{0}\right)$. Set $\left(\alpha_{0}, \beta_{0}\right)=(\gamma, \delta)$ and construct a self-financing strategy $\pi=\left(\alpha_{t}, \beta_{t}\right)_{t=0}^{T}$ and a stopping time $\sigma$ inductively as described in Theorem 3.3(i). In order to obtain (3.16) we show first that

$$
\left(\alpha_{\sigma}, \beta_{\sigma}\right) \in \operatorname{epi}\left(q_{\sigma}^{a}\right) .
$$

Indeed, if $\sigma=\sigma_{T}=t<T$ then by the construction $\sigma_{T}=\sigma_{T-1}=\cdots=\sigma_{t}, t-1<\sigma_{t-1}$ and $\left(\alpha_{t}, \beta_{t}\right) \in$ $\operatorname{epi}\left(q_{t}^{a}\right)$. If $\sigma=\sigma_{T}=T$ then we also obtain that

$$
\left(\alpha_{T}, \beta_{T}\right) \in \operatorname{epi}\left(q_{T}^{a}\right)
$$

since for otherwise by the construction we must have $\sigma_{T-1}=\sigma_{T}=T$. Then $T-1<\sigma_{T-1}$ and on this set (event) by construction $\left(\alpha_{T}, \beta_{T}\right) \in \operatorname{epi}\left(\mathbf{z}_{T-1}\right) \subset \operatorname{epi}\left(z_{T}\right)=\operatorname{epi}\left(q_{T}^{a}\right)$ since $z_{T}=r_{T}^{a}=q_{T}^{a}$, and so (4.2) holds true completing the proof of (4.1), as well. Since $G_{\sigma, t}=q_{\sigma}^{a}$ if $\sigma<t$ by (3.15) then (3.16) follows from (4.1) when $\sigma<t$. If $\sigma=t$ then $G_{\sigma, t}=r_{t}^{a}=r_{\sigma}^{a} \leq q_{\sigma}^{a}$, and so (4.1) implies (3.16) in this case, as well. Next, assume that $t<\sigma=\sigma_{T}$ and observe that by the construction $\{t<\sigma\} \subset\left\{t<\sigma_{t}\right\}$ for all $t=0,1, \ldots, T$. On $\left\{t<\sigma_{t}\right\}$ we have $\left(\alpha_{t}, \beta_{t}\right) \in \operatorname{epi}\left(z_{t}\right)$. But by (3.20) either $z_{t}=q_{t}^{a} \geq r_{t}^{a}$ or $z_{t}=\max \left(r_{t}^{a}, w_{t}\right) \geq r_{t}^{a}$ and in both cases $\left(\alpha_{t}, \beta_{t}\right) \in \operatorname{epi}\left(r_{t}^{a}\right)$ which yields (3.16) since $G_{\sigma, t}=r_{t}^{a}$ when $t<\sigma$.

Next, suppose that (b) holds true. Then (c) follows since (3.16) is equivalent to the seller's superhedging condition (2.5).

Suppose now that $(\sigma, \pi), \sigma \in \mathcal{T}, \pi=\left(\alpha_{t}, \beta_{t}\right)_{t=0}^{T}$ is a superhedging strategy for the seller such that $\left(\alpha_{0}, \beta_{0}\right)=(\gamma, \delta)$. Then (3.16) holds true. We prove next by the backward induction that

$$
\left(\alpha_{\sigma \wedge t}, \beta_{\sigma \wedge t}\right) \in \operatorname{epi}\left(z_{\sigma \wedge t}\right) \text {. }
$$

Observe that either $G_{\sigma, T}=r_{T}^{a}=z_{T}$ if $\sigma=T$ or $G_{\sigma, T}=q_{\sigma}^{a} \geq z_{\sigma}$ if $\sigma<T$. In both cases epi $\left(G_{\sigma, T}\right) \subset$ $\operatorname{epi}\left(z_{\sigma \wedge T}\right)$ and (4.3) with $t=T$ follows from (3.16). Now suppose that (4.3) holds true for some $t \in$ 
$\{1,2, \ldots, T\}$, i.e. $\left(\alpha_{t}, \beta_{t}\right) \in \operatorname{epi}\left(z_{t}\right)$. Since $\left(\alpha_{\sigma \wedge t}, \beta_{\sigma \wedge t}\right)$ is $\mathcal{F}_{\sigma \wedge(t-1)}$-measurable it follows by (3.20) that $\left(\alpha_{\sigma \wedge t}, \beta_{\sigma \wedge t}\right) \in$ epi $\left(\mathbf{z}_{\sigma \wedge(t-1)}\right)$. Because the strategy is self-financing, we have by (3.11) that

$$
\left(\alpha_{\sigma \wedge(t-1)}-\alpha_{\sigma \wedge t}, \beta_{\sigma \wedge(t-1)}-\beta_{\sigma \wedge t}\right) \in \operatorname{epi}\left(h_{\left[S_{\sigma \wedge(t-1)}^{b}, S_{\sigma \wedge(t-1)}^{a}\right]}\right),
$$

obtaining by (3.20) that

$$
\left(\alpha_{\sigma \wedge(t-1)}, \beta_{\sigma \wedge(t-1)}\right) \in \operatorname{epi}\left(h_{\left[S_{\sigma \wedge(t-1)}^{b}, S_{\sigma \wedge(t-1)}^{a}\right.}\right)+\operatorname{epi}\left(\mathbf{z}_{\sigma \wedge(t-1)}\right)=\operatorname{epi}\left(w_{\sigma \wedge(t-1)}\right) .
$$

Furthermore, by the superhedging condition (3.16),

$$
\left(\alpha_{\sigma \wedge(t-1)}, \beta_{\sigma \wedge(t-1)}\right) \in \operatorname{epi}\left(G_{\sigma, t-1}\right),
$$

and so,

$$
\begin{gathered}
\left(\alpha_{\sigma \wedge(t-1)}, \beta_{\sigma \wedge(t-1)}\right) \in \operatorname{epi}\left(\max \left(G_{\sigma, t-1}, w_{\sigma \wedge(t-1)}\right)\right) \\
\subset \operatorname{epi}\left(\max \left(r_{\sigma \wedge(t-1)}^{a}, w_{\sigma \wedge(t-1)}\right)\right) \subset \operatorname{epi}\left(z_{\sigma \wedge(t-1)}\right)
\end{gathered}
$$

since $z_{\sigma \wedge(t-1)} \leq \max \left(r_{\sigma \wedge(t-1)}^{a}, w_{\sigma \wedge(t-1)}\right)$ by (3.20) , completing the induction step. Now taking $t=0$ in (4.3) we obtain the assertion (a) proving the statement (i).

(ii) Suppose that $(\gamma, \delta) \in \operatorname{epi}\left(u_{0}\right)$. Set $\left(\alpha_{0}, \beta_{0}\right)=(\gamma, \delta)$ and construct a self-financing strategy $\pi=$ $\left(\alpha_{t}, \beta_{t}\right)_{t=0}^{T}$ and a stopping time $\tau$ inductively as described in Theorem 3.3(ii). In order to obtain (3.17) we show first that

$$
\left(\alpha_{\tau}, \beta_{\tau}\right) \in \operatorname{epi}\left(r_{\tau}^{b}\right)
$$

Indeed, if $\tau=\tau_{T}=t<T$ then by the construction $\tau_{T}=\tau_{T-1}=\cdots=\tau_{t}, t-1<\tau_{t-1}$ and $\left(\alpha_{t}, \beta_{t}\right) \in$ $\operatorname{epi}\left(r_{t}^{b}\right)$. If $\tau=\tau_{T}=T$ then we also obtain that

$$
\left(\alpha_{T}, \beta_{T}\right) \in \operatorname{epi}\left(r_{T}^{b}\right)
$$

since for otherwise by the construction we must have $\tau_{T-1}=\tau_{T}=T$. Then $T-1<\sigma_{T-1}$ and on this set (event) by the construction $\left(\alpha_{T}, \beta_{T}\right) \in \operatorname{epi}\left(\mathbf{u}_{T-1}\right) \subset \operatorname{epi}\left(u_{T}\right)=\operatorname{epi}\left(r_{T}^{b}\right)$ since $u_{T}=r_{T}^{b}$, and so (4.5) holds true completing the proof of (4.4), as well. Since $H_{s, \tau}=r_{\tau}^{b}$ if $\tau \leq s$ by (3.15) then (3.17) follows from (4.4) when $\tau \leq s$. Next, assume that $s<\tau=\tau_{T}$ and observe that by the construction $\{s<\tau\} \subset\left\{s<\tau_{s}\right\}$ for all $s=0,1, \ldots, T$. On $\left\{s<\tau_{s}\right\}$ we have $\left(\alpha_{s}, \beta_{s}\right) \in \operatorname{epi}\left(u_{s}\right)$. But $u_{s} \geq q_{s}^{b}$ by (3.22), and so $\left(\alpha_{s}, \beta_{s}\right) \in$ epi $\left(q_{s}^{b}\right)$ on $\left\{s<\tau_{s}\right\}$, which yields (3.17) since $H_{s, \tau}=q_{s}^{b}$ when $s<\tau$.

Next, suppose that (e) holds true. Then (f) follows since (3.17) is equivalent to the buyer's superhedging condition (2.8).

Suppose now that $(\tau, \pi), \tau \in \mathcal{T}, \pi=\left(\alpha_{t}, \beta_{t}\right)_{t=0}^{T}$ is a superhedging strategy for the buyer such that $\left(\alpha_{0}, \beta_{0}\right)=(\gamma, \delta)$. Then (3.17) holds true. We prove next by the backward induction that

$$
\left(\alpha_{s \wedge \tau}, \beta_{s \wedge \tau}\right) \in \operatorname{epi}\left(u_{s \wedge \tau}\right) .
$$

Observe that either $H_{T, \tau}=r_{T}^{b}=u_{T}$ if $\tau=T$ or $H_{T, \tau}=r_{\tau}^{b} \geq u_{\tau}$ if $\tau<T$. In both cases epi $\left(H_{T, \tau}\right) \subset$ epi $\left(u_{T \wedge \tau}\right)$ and (4.6) with $s=T$ follows from (3.17). Now suppose that (4.6) holds true for some $s \in$ $\{1,2, \ldots, T\}$, i.e. $\left(\alpha_{s}, \beta_{s}\right) \in \operatorname{epi}\left(u_{s}\right)$. Since $\left(\alpha_{s \wedge \tau}, \beta_{s \wedge \tau}\right)$ is $\mathcal{F}_{(s-1) \wedge \tau}$-measurable it follows by (3.22) that $\left(\alpha_{s \wedge \tau}, \beta_{s \wedge \tau}\right) \in \operatorname{epi}\left(\mathbf{u}_{(s-1) \wedge \tau}\right)$. Because the strategy is self-financing, we have by (3.11) that

$$
\left(\alpha_{(s-1) \wedge \tau}-\alpha_{s \wedge \tau}, \beta_{(s-1) \wedge \tau}-\beta_{s \wedge \tau}\right) \in \operatorname{epi}\left(h_{\left[S_{(s-1) \wedge \tau}^{b}, S_{(s-1) \wedge \tau}^{a}\right]}\right),
$$

obtaining by (3.22) that

$$
\left(\alpha_{(s-1) \wedge \tau}, \beta_{(s-1) \wedge \tau}\right) \in \operatorname{epi}\left(h_{\left[S_{(s-1) \wedge \tau}^{b}, S_{(s-1) \wedge \tau}^{a}\right]}\right)+\operatorname{epi}\left(\mathbf{u}_{(s-1) \wedge \tau}\right)=\operatorname{epi}\left(v_{(s-1) \wedge \tau}\right) .
$$

Furthermore, by the superhedging condition (3.17),

$$
\left(\alpha_{(s-1) \wedge \tau}, \beta_{(s-1) \wedge \tau}\right) \in \operatorname{epi}\left(H_{s-1, \tau}\right),
$$


and so,

$$
\begin{gathered}
\left(\alpha_{(s-1) \wedge \tau}, \beta_{(s-1) \wedge \tau}\right) \in \operatorname{epi}\left(\max \left(H_{s-1, \tau}, v_{(s-1) \wedge \tau}\right)\right) \\
\subset \operatorname{epi}\left(\max \left(q_{(s-1) \wedge \tau}^{b}, v_{(s-1) \wedge \tau}\right)\right) \subset \operatorname{epi}\left(u_{(s-1) \wedge \tau}\right)
\end{gathered}
$$

since $H_{s-1, \tau} \geq q_{(s-1) \wedge \tau}^{b}$ and $u_{(s-1) \wedge \tau} \leq \max \left(q_{(s-1) \wedge \tau}^{b}, v_{(s-1) \wedge \tau}\right)$ by (3.22), completing the induction step. Now taking $s=0$ in (4.6) we obtain the assertion (d) proving the statement (ii).

Now we are ready to prove Theorems 3.1]3.3. In view of Lemma 4.1(i) it follows from (2.7) that

$$
\begin{gathered}
V^{a}=\min \left\{-\theta_{0}(-\gamma,-\delta):(\gamma, \delta) \in \operatorname{epi}\left(z_{0}\right)\right\} \\
\leq \min \left\{-\theta_{0}(-\gamma, 0):(\gamma, 0) \in \operatorname{epi}\left(z_{0}\right)\right\} \leq \min \left\{\gamma:(\gamma, 0) \in \operatorname{epi}\left(z_{0}\right)\right\}=z_{0}(0) .
\end{gathered}
$$

In order to derive the inequality in the other direction we will use $z_{\sigma, s}, s=0,1, \ldots, T$ constructed in Theorem 3.2 (i) for any $\sigma \in \mathcal{T}$. First, we show by the backward induction that for all $s=0,1, \ldots, T$,

$$
z_{\sigma, s} \geq z_{\sigma \wedge s} .
$$

Indeed, for $s=T$ we have $z_{\sigma, T}=G_{\sigma, T} \geq z_{\sigma \wedge T}$ which is clear from the definitions when $\sigma=T$ while if $\sigma<T$ then $z_{\sigma, T}=q_{\sigma}^{a} \geq z_{\sigma}$. Suppose that (4.8) holds true for $s=T, T-1, \ldots, t$. In order to prove it for $s=t-1$ we observe that by (3.20),

$$
z_{\sigma, t-1} \geq G_{\sigma, t-1}=q_{\sigma}^{a} \geq z_{\sigma}=z_{\sigma \wedge(t-1)}
$$

on the set $\{\sigma<t-1\}$. On the other hand, on the set $\{\sigma \geq t-1\}$ by the definition and the induction hypothesis $\mathbf{z}_{\sigma, \mathbf{t}-\mathbf{1}} \geq \mathbf{z}_{\mathbf{t}-\mathbf{1}}$. This together with the monotonicity of the operator $\operatorname{gr}_{[d, c]}$ yields that $w_{\sigma, t-1} \geq$ $w_{t-1}$. Hence, on $\{\sigma \geq t-1\}$ by (3.20),

$$
z_{t-1}^{\mu} \leq \max \left(r_{t-1}^{a}(\mu, x), w_{t-1}^{\mu}(x)\right) \leq \max \left(G_{\sigma, t-1}(\mu, r), w_{\sigma, t-1}^{\mu}\right)=z_{\sigma, t-1}^{\mu}
$$

completing the induction step, and so (4.8) is valid for all $s=0,1, \ldots, T$.

For each $\sigma \in \mathcal{T}$ set

$$
V_{\sigma}^{a}=\inf _{\pi}\left\{-\theta_{0}\left(-\alpha_{0},-\beta_{0}\right):(\sigma, \pi) \text { with } \pi=\left(\alpha_{t}, \beta_{t}\right)_{t=0}^{T} \text { being a superhedging strategy for the seller }\right\} .
$$

It is easy to see that $V_{\sigma}^{a}$ is the seller's (ask) price of the American option with the payoff process $Q_{\sigma, t}=\left(Q_{\sigma, t}^{(1)}, Q_{\sigma, t}^{(2)}\right), t=0,1, \ldots, T$ (see (2.4) $)$ and the same transactions costs setup as before. Hence, we can rely on Theorem 3.3 from $[8$ in order to claim that

$$
\begin{gathered}
V_{\sigma}^{a}=\max _{\chi \in \mathcal{X}} \max _{(P, S) \in \overline{\mathcal{P}}(\chi)} \mathbb{E}_{P}\left(Q_{\sigma, \cdot}^{(1)}+S Q_{\sigma, \cdot}^{(2)}\right)_{\chi} \\
=\max _{\chi \in \mathcal{X}} \sup _{(P, S) \in \mathcal{P}(\chi)} \mathbb{E}_{P}\left(Q_{\sigma, \cdot}^{(1)}+S Q_{\sigma, \cdot}^{(2)}\right)_{\chi}=z_{\sigma, 0}(0) .
\end{gathered}
$$

Combining (2.7), (4.8) and (4.10) we obtain that

$$
\begin{gathered}
V^{a}=\min _{\sigma \in \mathcal{T}} V_{\sigma}^{a}=\min _{\sigma \in \mathcal{T}} \max _{\chi \in \mathcal{X}} \max _{(P, S) \in \overline{\mathcal{P}}(\chi)} \mathbb{E}_{P}\left(Q_{\sigma, \cdot}^{(1)}+S Q_{\sigma, \cdot}^{(2)}\right)_{\chi} \\
=\min _{\sigma \in \mathcal{T}} \max _{\chi \in \mathcal{X}} \sup _{(P, S) \in \mathcal{P}(\chi)} \mathbb{E}_{P}\left(Q_{\sigma, \cdot}^{(1)}+S Q_{\sigma, \cdot}^{(2)}\right)_{\chi}=\min _{\sigma \in \mathcal{T}} z_{\sigma, 0}(0) \geq z_{0}(0) .
\end{gathered}
$$

Now (4.7) together with (4.11) yields both (3.9) from Theorem 3.1] and the conclusion of Theorem 3.2(i).

It follows from the equality $V^{a}=z_{0}(0)$ that $\left(V^{a}, 0\right) \in \operatorname{epi}\left(z_{0}\right)$. On the other hand, we showed already in the proof of Lemma 4.1(i) that any pair $(\sigma, \pi)$ of $\sigma \in \mathcal{T}$ and $\pi=\left(\alpha_{t}, \beta_{t}\right)_{t=0}^{T} \in \Phi$ with $\left(\alpha_{0}, \beta_{0}\right) \in \operatorname{epi}\left(z_{0}\right)$ constructed by the algorithm of Theorem $3.3(\mathrm{i})$ is a superhedging strategy for the seller. Hence, the conclusion of Theorem 3.3(i) follows, completing the proof of our results concerning the seller.

In order to obtain our results concerning the buyer we will rely on the duality of the seller and the buyer positions in the setup of game options. Indeed, since negative payoffs are also allowed we can view now the buyer as a seller of the new game option with the payoff (2-vector) function

$$
\hat{Q}_{s, t}=\left(\hat{Q}_{s, t}^{(1)}, \hat{Q}_{s, t}^{(2)}\right)=-X_{s} \mathbb{I}_{s<t}-Y_{s} \mathbb{I}_{t \leq s}=-Q_{s, t}
$$


while the former seller becomes a buyer of this new option. We observe the slight difference here that when $s=t$ the payoff $-Y_{s} \geq-X_{s}$ includes "the penalty" but this convention does not influence the results. Now we see that replacing $Q_{s, \tau}^{(1)}$ and $Q_{s, \tau}^{(2)}$ by $\hat{Q}_{s, \tau}^{(1)}$ and $\hat{Q}_{s, \tau}^{(2)}$ in the superhedging condition (2.8) transforms it into the form (2.5) and writing (2.8) for $\hat{V}^{b}=-V^{b}$ we transform (2.8) into the form (2.7). Next, $H_{s, t}$ in (3.15) will have the form of $G_{s, t}$ there if we replace $Q_{s, t}^{(1)}$ and $Q_{s, t}^{(2)}$ there by $\hat{Q}_{s, t}^{(1)}$ and $\hat{Q}_{s, t}^{(2)}$, respectively. Furthermore, (3.10) will have the form of (3.9) if we rewrite it for $\hat{V}^{b}, \hat{Q}_{\cdot, \tau}^{(1)}, \hat{Q}_{\cdot, \tau}^{(2)}$ in place of $V^{b}, Q_{\cdot, \tau}^{(1)}, Q_{\cdot, \tau}^{(2)}$, respectively. Taking into account these arguments we derive (3.10) and the assertions (ii) of Theorems 3.2 and 3.3 from (3.9) and from the corresponding assertions (i) of these theorems.

\section{Shortfall Risk AND PARTIAL HedGing}

In this section we discuss the shortfall risk for game options with transaction costs. The shortfall risk of the seller using a cancellation stopping time $\sigma$ and a self-financing strategy $\pi=\left(\alpha_{t}, \beta_{t}\right)_{t=0}^{T}$ is defined by

$$
R^{a}(\sigma, \pi)=\sup _{\tau \in \mathcal{T}} \mathbb{E}_{\mathbb{P}}\left(\theta_{\sigma \wedge \tau}\left(\alpha_{\sigma \wedge \tau}-Q_{\sigma, \tau}^{(1)}, \beta_{\sigma \wedge \tau}-Q_{\sigma, \tau}^{(2)}\right)\right)^{-}
$$

where $a^{-}=-\min (a, 0)$. The shortfall risk of the seller wishing to spend no more than an amount $x$ in order to set his (partial) hedging portfolio is defined by

$$
R^{a}(x)=\inf _{\sigma \in \mathcal{T}, \pi}\left\{R(\sigma, \pi): \pi=\left(\alpha_{t}, \beta_{t}\right)_{t=0}^{T} \text { is self-financing and }-\theta_{0}\left(-\alpha_{0},-\beta_{0}\right) \leq x\right\} .
$$

Similarly, we can define the shortfall risk of the buyer using an exercise stopping time $\tau$ and a selffinancing portfolio strategy $\pi=\left(\alpha_{t}, \beta_{t}\right)_{t=0}^{T}$ by

$$
R^{b}(\tau, \pi)=\sup _{\sigma \in \mathcal{T}} \mathbb{E}_{\mathbb{P}}\left(\theta_{\sigma \wedge \tau}\left(\alpha_{\sigma \wedge \tau}+Q_{\sigma, \tau}^{(1)}, \beta_{\sigma \wedge \tau}+Q_{\sigma, \tau}^{(2)}\right)\right)^{-}
$$

where $a^{-}=-\min (a, 0)$. The shortfall risk of the buyer who takes a loan in the amount at least $x$ to pay it for the option and to set a (partial) hedging portfolio is defined by

$$
R^{b}(x)=\inf _{\tau \in \mathcal{T}, \pi}\left\{R^{b}(\tau, \pi): \pi=\left(\alpha_{t}, \beta_{t}\right)_{t=0}^{T} \text { is self-financing and } \theta_{0}\left(-\alpha_{0},-\beta_{0}\right) \geq x\right\} .
$$

The shortfall risk of the buyer measures the maximal expected amount which may be needed to settle buyer's bank loan in addition to his portfolio liquidation value and the option payoff he receives from the seller.

Observe that usually $V^{a}>V^{b}$ and in this case if the buyer agrees to pay for the option the upper hedging price $V^{a}$ (or any price higher than $V^{b}$ ) he should take into account the shortfall risk while the same is true concerning the seller if he agrees to sell the option for the lower hedging price (or any price lower than $V^{a}$ ). It is not difficult to see that in the study of the shortfall risk pure stopping times suffice and there is no need to deal with randomized ones which would lead actually to the same values of the shorfall risk in view of linearity of corresponding expressions. Some dynamical programming algorithms for computation of shortfall risks and corresponding partial hedging strategies can be constructed for game options with proportional transaction costs in finite discrete markets similarly to $[\underline{3}$ where such algorithms were described for binomial models of American options but their extension to game options is also possible (see Remark 6.3 in [3]) which was done for frictionless multinomial markets in [5].

\section{REFERENCES}

[1] B. Bouchard and E. Temam, On the hedging of American options in discrete time markets with proportional transaction costs, Electron. J. Probab. 10 (2005), 746-760.

[2] P. Chalasani and S. Jha, Randomized stopping times and American option pricing with transaction costs, Math. Finance 11 (2001), 33-77.

[3] Ya. Dolinsky, Limit theorems for partial hedging under transaction costs, Math. Finance, to appear.

[4] Ya. Dolinsky, Hedging of game options with the presence of transaction costs, Ann. Appl. Probab., to appear. 
[5] Ya. Dolinsky and Yu. Kifer, Hedging with risk for game options in discrete time, Stochastics 79 (2007), $169-195$.

[6] Yu. Kifer, Game options, Finance and Stoch. 4 (2000), 443-463.

[7] R.T. Rockafeller, Convex Analysis, Princeton Univ. Press, Princeton (1997).

[8] A. Roux and T. Zastawniak, American options under proportional transaction costs: pricing, hedging and stopping algorithms for long and short positions, Acta Appl. Math. 106 (2009), 199-228.

Institute of Mathematics, The Hebrew University, Jerusalem 91904, Israel

E-mail address: kifer@math.huji.ac.il 